Case report

\title{
The use of fulvestrant in patients with advanced breast cancer
}

\section{Joanna Kufel-Grabowska}

Greater Poland Centre of Oncology in Poznań

Correspondence: Joanna Kufel-Grabowska Greater Poland Centre of Oncology in Poznań 61-866 Poznań, ul. Garbary 15 e-mail:joannakufel@googlemail.com

Received: 6.02.2018. Accepted: 23.03.2018.

DOI: 10.24292/01.OR.230318 Copyright $\odot$ Medical Education. All rights reserved.

\section{ABSTRACT}

Breast cancer is the most common malignancy in women in Poland and the entire world, but thanks to early diagnostics and efficacious therapeutic methods, its prognosis is good. In some patients with advanced breast cancer, long-term survival is observed, which is why it is essential to select the most appropriate therapy, and to follow up on the course of disease and possible adverse events related to the treatment applied. The slow development of hormone-receptor-positive cancer, and a multitude of therapeutic options constitute a real challenge in palliative treatment. The use of fulvestrant resulted in a good response in both of the presented cases, and did not in any way comprise the daily lives of the patients over a long period of time.

Key words: breast cancer, fulvestrant, hormone therapy 


\section{INTRODUCTION}

Breast cancer is one of the most common malignancies in women in Poland and the entire world. Mortality due to breast cancer has given way to mortality related to lung cancer, which is directly linked to an increase in the popularity of tobacco smoking amongst women [1]. Early detection of breast cancer, followed by appropriate treatment, makes it possible to cure most patients now. Some women are diagnosed with advanced disease, though, or experience cancer recurrence despite previous radical therapy. However, even in those patients, it is sometimes possible to achieve long-term survival. Thus, it is essential to plan the treatment of that slowly progressing disease carefully, closely monitoring the course of disease as well the possible adverse events related to the therapy administered and to patient comorbidities.

The most aggressive biological subtypes of breast cancer include the triple negative breast cancer, showing no expression of steroid receptors and no HER2 receptor overexpression, and the HER2-positive cancer. In the event of recurrence of the triple negative breast cancer, chemotherapy is the treatment of choice, whereas if there is a relapse of the HER2-positive cancer, targeted therapy is of paramount importance, directed at the HER2 receptor. Presently, there are two molecules available in Poland, trastuzumab and pertuzumab, as well as a tyrosine kinase inhibitor lapatinib.

Most breast cancers show expression of oestrogen and/or progesterone receptors. The main source of steroid hormones are ovaries in pre-menopausal women, and peripheral tissues in post-menopausal women, including the adipose tissue, muscles and breasts. In light of the novel therapies of hormone-dependent tumours, it is a real challenge to decide on an adequate sequence of hormonal medications, and chemotherapy, if needs be. The role of hormone treatment is to restrict the effects of oestrogens on their target receptor.

Tamoxifen plays an essential part in the treatment of both preand post-menopausal female patients. Its action blocks the oestrogen receptor in breast tissues, inhibiting cancer recurrence, and lowering the risk of cancer development in the contralateral breast. At the same time, the drug stimulates the oestrogen receptor in endometrium, thus increasing the risk of neoplasia in the uterine cavity. On the other hand, it also stimulates bone formation and the cardiovascular system. Due to the different effects on the oestrogen receptor in different locations, inhibitory or excitatory, tamoxifen belongs to a group of medicines known as selective oestrogen receptor modulators [2]. A drug that only has an inhibitory effect is fulvestrant, which competitively inhib- its the binding of oestradiol with the oestrogen receptor, leading to its degradation. Progression of cancer despite the use of fulvestrant may testify to the presence of resistance mechanisms, and to the development of independent hormone stimulation pathways [3].

Aromatase inhibitors are mainly used in post-menopausal patients. Their role consists in inhibiting aromatization of androgens into oestrogens in the adipose tissue, which constitutes the primary source of sex hormones in post-menopausal women.

When selecting hormone therapies, the sequence of therapeutics depends on the advancement of tumour, the patient's general condition and concomitant diseases. Even in metastatic disease, without severe symptoms, hormone treatment is possible, with chemotherapy reserved primarily for organ crises or rapid disease progression [4].

The below descriptions of the course of disease in two of our patients testify to a high and long-lasting efficacy of the hormone therapies involved.

\section{CASE 1.}

In September 2016, a 62-year-old female patient was referred for consultation at the Chemotherapy Clinic of the Greater Poland Centre of Oncology due to the dissemination of cancer cells into the peritoneal cavity. In 2000, she had undergone left-sided mastectomy for steroid receptor-positive HER2-negative lobular breast cancer. She had received the $A C$ chemotherapy regimen (4 courses of doxorubicin cyclophosphamide) and tamoxifen. 3 years from the completion of hormone therapy, lower back pain emerged in the patient. X-ray examination was performed, followed by bone scintigraphy, which revealed metastatic lesions in the thoracic and lumbar vertebrae as well as within the sternum and the ribs. Additional imaging tests did not reveal any significant abnormalities, but the Ca 15.3 marker continued to be elevated. At that time, the patient was started on tamoxifen plus sodium pamidronate as well as vitamin D and calcium supplementation. 2 years into the treatment, the back pains were exacerbated, imaging tests revealed disease progression, and the $\mathrm{Ca}$ 15.3 level was elevated. In 2010, letrozole was initiated, alongside the continued bisphosphonate therapy. For the following 5 years, the patient was under oncological follow-up, and continued receiving the previously prescribed medications. In 2015, further progression of bone lesions was observed, accompanied by periodic back and pelvic pain. When letrozole was replaced by exemestane, and pamidronate was changed to zoledronic acid, 
the pain receded, and the Ca 15.3 tumour marker concentration decreased. Due to the long-standing bisphosphonate use and a normal blood calcium level, it was recommended that zoledronic acid be administered every 12 weeks.

In May 2016, the patient underwent an urgent surgery at the regional hospital for abdominal pain. The surgery confirmed intestinal obstruction, and a fragment of the intestine with lobular cancer infiltration was resected. Subsequently, the patient was referred for further oncological diagnostics. The test performed revealed a significant increase in the Ca 15.3 tumour marker level to $174.4 \mathrm{U} / \mathrm{ml}$ (normal range: $15-30 \mathrm{U} / \mathrm{ml}$ ), metastatic lesions involving the right ovary, and some slight urinary retention in the right kidney. The patient was qualified for the removal of the reproductive organs. In September 2016, she underwent radical hysterectomy, with the resection of peritoneal neoplastic lesions. Following the surgery, slight urinary stasis in the right kidney persisted, caused by the spread of the tumour within the peritoneal cavity. In November 2016, the patient was qualified for cytostatic treatment in line with the MC regimen (non-pegylated liposomal doxorubicin, cyclophosphamide). Echocardiography examination was also performed, revealing left ventricular ejection fraction (LVEF) of $63 \%$. The patient received 7 courses of chemotherapy, with the urinary stasis symptoms receding after the first 3 courses. Throughout the therapy, a systematic drop of the tumour marker was observed, down to the level of $47 \mathrm{U} / \mathrm{ml}$ and the LVEF value was normal. A total of $480 \mathrm{mg} / \mathrm{m}^{2}$ of liposomal doxorubicin was administered, and as the cancer symptoms resolved, it was decided to continue maintenance treatment with fulvestrant. The first dose was administered in April 2017, and the therapy has continued to this day. Since the beginning of January 2018, the Ca 15.3 tumour marker levels have been growing, which is why follow-up imaging tests have been scheduled. The patient's general condition has been good, though, and is not indicative of cancer progression.

\section{CASE 2.}

In July 2016, a 64-year-old female patient reported to the Chemotherapy Clinic of the Greater Poland Centre of Oncology due to dyspnoea and deterioration of her general condition. The symptoms had been intensifying for around a month. The patient had undergone left pleural puncture at the regional hospital, where ca. 3 litres of haemorrhagic effusion had been evacuated, leading to a temporary improvement of the patient's general condition. A cytology test performed at the time revealed no presence of cancer cells. Subsequently, the patient was urgently referred for oncological consultation.
The patient had a history of left breast cancer, detected in 1999, when a left-sided mastectomy was performed, followed by the adjunctive CMF chemotherapy (cyclophosphamide, methotrexate, fluorouracil), and a subsequent 5-year tamoxifen therapy. From then on, the patient had remained under oncological follow-up.

During the patient's stay at the Chemotherapy Department of the Greater Poland Centre of Oncology, fluid was removed from the left pleural cavity, followed by laboratory and imaging tests. What required attention was the significantly elevated concentration of the Ca 15.3 tumour marker (912 U/ml), infiltration of the left pleura and left thoracic wall, and the enlarged lymph nodes within the mediastinum and the abdominal cavity. Biopsy of the thoracic metastatic lesion (with primary tumour morphology) was performed, confirming steroid receptor-positive lobular breast cancer metastasis. Positron emission tomography (PET) scan revealed no additional foci showing signs of an active neoplastic process. In July, a further increase in the Ca 15.3 level was observed, bringing the tumour marker concentration up to $1440 \mathrm{U} / \mathrm{ml}$. Due to the intensified symptoms of the disease, the patient was qualified for cytostatic treatment based on the MC pattern (non-pegylated liposomal doxorubicin, cyclophosphamide). Her echocardiogram revealed the LVEF of $67 \%$. Following 7 chemotherapy courses, echocardiography tests were performed every 3 weeks, with no significant left ventricular contractility dysfunction observed. The patient received a total of 11 chemotherapy courses, completing the treatment in March 2017 , and subsequently moving on to fulvestrant therapy. She tolerated the chemotherapy very well, her dyspnoea resolved after 4 courses, and the Ca 15.3 tumour marker levels went back to normal towards the end of the treatment period. Presently, the patient is 12 months into her hormone therapy, she continues her professional career, and has a very good exercise tolerance.

\section{DISCUSSION}

In both of the presented patients, breast cancer was diagnosed a dozen or so years ago, and in the first case, the progression of metastatic disease has been a slow one. For 8 years, the disease has progressed very slowly, without significantly compromising her daily life, and enabling regular professional activities. Despite periodic deterioration of her general condition, effective chemotherapy made it possible for her to go back to her usual level of activity.

The second patient reported for treatment at a time of an organ crisis, requiring immediate chemotherapy, which turned out to be 
highly effective. Having completed the chemotherapy, the patient resumed her professional career, and expected further treatment to be as convenient as possible. The choice of fulvestrant was fully accepted by her, effectively supplementing prior treatment.

\section{CONCLUSIONS}

Breast cancer has become a chronic disease, and the two cases discussed above reflect the phenomenon very well. Fulvestrant is a drug that is active both following an earlier anti-oestrogen therapy as well as in the first line of dissemination, as confirmed by the Falcon study results. In the group of patients receiving fulvestrant, time to progression was 16.6 months, and was 2.8 months longer as compared with the anastrozole study arm (13.8 months) [5].

Treating breast cancer patients, we administer hormone therapy in bone and lymph node metastases, and ever more willingly also in organ metastases, with the presented two cases being a good example of the current trend.

\section{References}

1. Bojakowska U, Kalinowski P, Kowalska ME. Epidemiologia i profilaktyka raka piersi. J Educ Health Sport 2016; 6(8): 701-710.

2. Sporn MB, Lippman SM. Agents for Chemoprevention and Their Mechanism of Action. In: Kufe DW, Pollock RE, Weichselbaum RR et al. (ed). Holland-Frei Cancer Medicine. 6th edition. Hamilton (ON): BC Decker 2003.

3. Carlson RW. The history and mechanism of action of fulvestrant. Clin Breast Cancer 2005; 6(suppl 1): s5-s8.

4. Cardoso F, Costa A, Senkus E et al. 3rd ESO-ESMO International Consensus Guidelines for Advanced Breast Cancer (ABC 3). Ann Oncol 2017; 28(1): 16-33.

5. Robertson JFR, Bondarenko I, Trishkina E. Fulvestrant $500 \mathrm{mg}$ versus anastrozole $1 \mathrm{mg}$ for hormone receptor-positive advanced breast cancer (FALCON): an international, randomised, double-blind, phase 3 trial. Lancet 2017; 388(10063): 2997-3005. 